\title{
Satellite Navigation and Communication Integration Based on Correlation Domain Indefinite Pulse Position Modulation Signal
}

\author{
Deyue Zou (iD) and Shouchuan Ma \\ Dalian University of Technology, Dalian, China \\ Correspondence should be addressed to Deyue Zou; zoudeyue@dlut.edu.cn
}

Received 25 February 2021; Accepted 24 May 2021; Published 7 June 2021

Academic Editor: Weizh Meng

Copyright () 2021 Deyue Zou and Shouchuan Ma. This is an open access article distributed under the Creative Commons Attribution License, which permits unrestricted use, distribution, and reproduction in any medium, provided the original work is properly cited.

\begin{abstract}
Ubiquitous signal coverage is a basic demand of Internet of Things (IoT) communications, which meets the feature of satellite communications. Infinite user number is a basic demand of IoT location-based services, which meets the feature of Global Navigation Satellite System (GNSS). Both of these demands make Satellite Navigation and Communication Integration (SNCI) an important supporting technology for IoT. Inherited from the satellite communications system, GNSS itself has a certain data transmission capacity. Thus, enhancing the communication function of the GNSS is a promising means of achieving SNCI. Considering that a unified signal system cannot currently realize high-precision positioning and high-speed data transmission simultaneously in SNCI, this project proposes a Correlation Domain Indefinite Pulse Position Modulation (CDIPPM). A pilot channel and a data channel are introduced in this technology, which are distinguished by Code Division Multiplexing (CDMA). The synchronization function is provided by the pilot channel, thereby freeing the data channel of this function. The phase of the pseudorandom code can then be used as the carrier of information. In order to transmit more information, the transmitter of the proposed technology superimposes on the data channel multiple sets of spread spectrum sequence, which are generated from one set of spread spectrum sequence by different cyclic shifting operations. The receiver will identify the number and location of the correlation function peaks by a detection algorithm and recover the message. It can be seen by theoretical analysis and simulation verification. The technology can significantly improve satellite data transmission rates and maintain the original positioning function while minimizing change in the original GNSS signal. Therefore, the SNCI system based on this technology has the following advantages: a unified signal system, high positioning accuracy, high data transmission rate, and a backward navigation function, and it is easy to promote.
\end{abstract}

\section{Introduction}

The idea of SNCI has been explored in previous studies. Both Globalstar [1,2] and BeiDou systems have the navigation capabilities as well as communication services. In the recent search for the missing Malaysian Airlines flight MH370 [3], Rolls Royce provided a part of flight path. This path is estimated from the working condition monitoring signal sent by the engine, which is based on the same principle as GNSS.

Through analyzing the status quo of SNCI technology, it can be found that the current SNCI research adopts three main methods. The first one is sending data to users through an independent satellite communications system. In order to ensure the consistency of terminals, this kind of satellite sys- tem tends to use the same frequency points and modulation modes as the GNSS, and its information rates are also consistent with the corresponding GNSS. Typical instances of this method include the WAAS $[4,5]$ of the United States, the European EGNOS system [6], the Japanese MSAS [7, 8], Japanese LEX signal $[9,10]$, the Indian GAGAN system [11-13], and the Russian SDCM system [14]. Therefore, these systems usually have a lower information rate, which is generally 250 bps. With the growing demand for improvement of the users' communication capability, most countries have begun to use common communication satellite systems to send navigation enhancement information. This solution increases the transmission rate but sacrifices the unity of receiving terminals, which is supposed to be the technical advantage of SNCI 


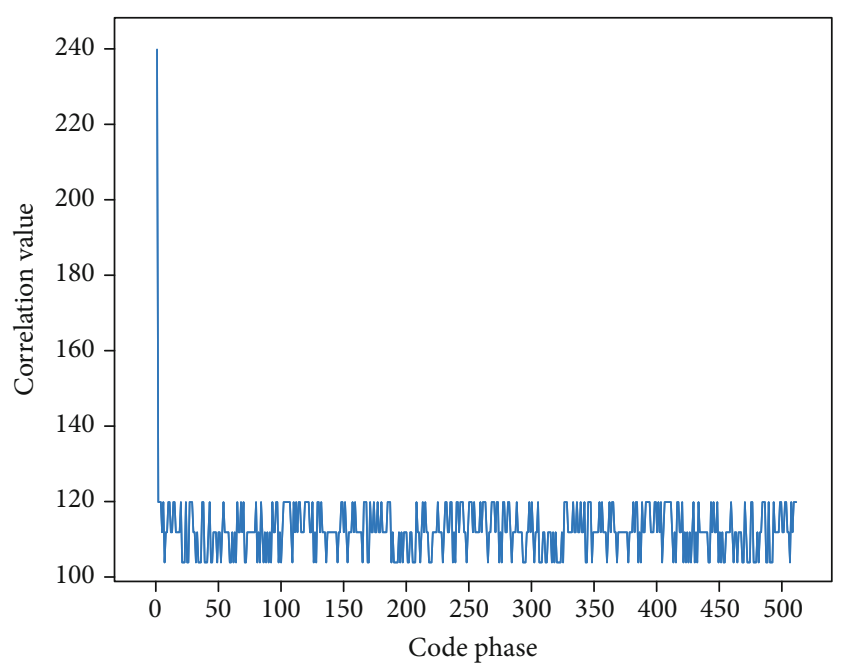

(a) Synchronized

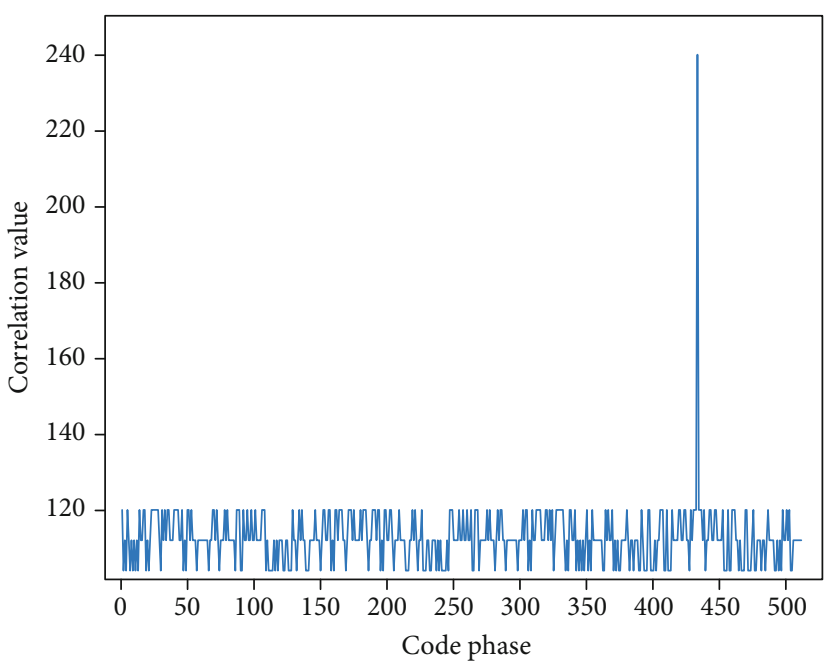

(b) Cyclically shifted

FIGURE 1: The autocorrelation function of a Gold sequence: a big correlation value can be calculated when the received sequence and local sequence are synchronized. The position of the correlation peak was changed when the received sequence is cyclically shifted.

systems. Additionally, this method has deviated from the initial purpose of SNCI technology. A typical case is the HI-GPS project of Boeing in 2008, supported by the US Army, which utilized the Iridium satellite system. Similarly, the newly introduced Hongyan small satellite system of China also includes the positioning enhancement function in its plan.

The second method to achieve SNCI is by promoting the range capabilities of the communication signal. However, the key challenges of communication signal design are increasing the data rate and the channel quantity while navigation signals are more focused on range accuracy. Due to the different design requirements, a single signalling system must compromise between the two aspects or be more focused on one of them. For example, the Globalstar system and the Inmarsat $[15,16]$ system of the US have strong communication capabilities but limited navigational precision and continuity. China's BeiDou system provides a different example where there is strong positioning capability but low information transfer rate. By adopting the RDSS positioning scheme, the amount of data dedicated to navigation services is reduced in the BeiDou system, leaving more resources for carrying communication information in each data frame. In addition, the CAPS [17] system proposed by CAAS National Astronomical Observatories in 2002 also belongs to this type of SNCI system [18].

The third method is to combine communication and navigation functions mechanically on the same platform. At present, the attempts by industry mostly fall into this category. However, in this category, navigation and communication functions are implemented independently without deep integration. No matter whether the communication system is set solely for GNSS or bundles two hardware devices together mechanically, the spectrum resources are used independently.

Therefore, compared with the first and third methods, the second one is more inclined to achieve deep integration of navigation and communication functions on the signal layer. Since the signal pattern of communication satellites may not be able to meet the requirements of timing and ranging, many of the ideas for SNCI are based on existing navigation satellite systems. This method effectively utilizes the existing satellite resources and reduces the construction cost. However, the communication rate of these systems is low because they are limited by insufficient band frequency and energy resources. In the future construction of SNCI, the constellation and spectrum conditions may undergo great changes, and they may not be limited by the states of the current systems. As mentioned above, communication satellite systems are converging with GNSS in constellation and signal characteristics while the frequency resources are insufficient in the satellite-ground link. For this reason, the operational bandwidth of navigation satellites and communication satellites has become very close, e.g., both Inmarsat $[19,20]$ and GNSS satellites operate on the L-band. This would allow the two systems to share their existing spectrum resources for stronger communication and positioning capabilities if they were unified in the future [21].

Furthermore, compared with most of the modern communication systems $[22,23]$, the transmit speed of GNSS is too slow. This brought the latency, vulnerability, and low adaptability to the scene. Many researches are focused in these problems [24-30], and increasing the transmit speed problem of GNSS would be helpful to these problems.

In this paper, a method of CDIPPM is proposed. This method uses the navigation signal as the pilot channel, so that the pseudonoise $(\mathrm{PN})$ sequence on the data channel can carry information by code phase. Furthermore, an indefinite amount of data channels is combined together to increase the amount of information that is carried by each symbol. Thus, the communication rate of the satellite downlink signal is improved.

The structure of this paper is as follows. The second part introduces the basic principles of CDIPPM. The third part describes the signal transmission strategy of the proposal. 


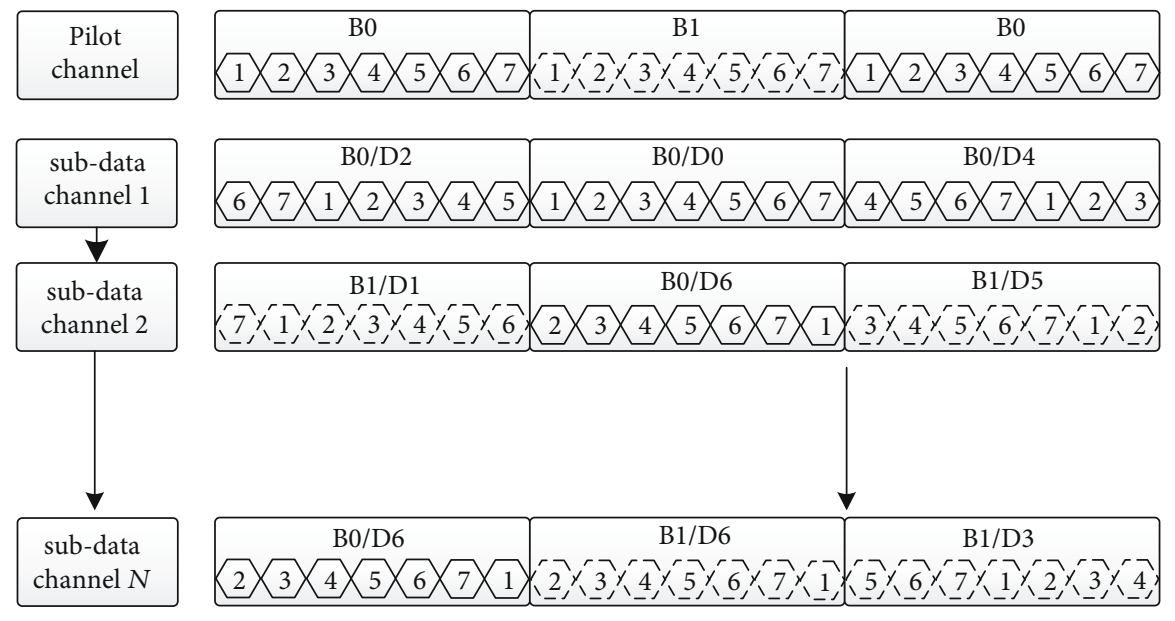

FIgURE 2: The structure of CDIPPM signal: the number of subdata channels is indefinite.

The system performance of this technology is analyzed and verified in the fourth and fifth parts. The conclusion follows those parts.

\section{Basic Principle of CDIPPM}

In Direct Sequence Spread Spectrum (DSSS), correlation operations are used for signal detection. At the same time, due to the good autocorrelation of the spreading sequence, the system also ensures the bit synchronization of the signals in the process of detection. The correlation property of the $\mathrm{PN}$ sequence is such that if and only if the received sequence is synchronized with the local sequence, a peak appears in the correlation function and the receiver establishes the bit synchronization. During the rest of the time, the correlation functions are at smaller values, as shown in Figure 1(a). Under the bit-synchronized state, if the PN sequence is cyclically shifted by $s$ bits and then transmitted, the peak would be found at $s$ position in the correlation function, as shown in Figure 1(b).

With this feature, the receiving equipment can demodulate the information that was carried by the code phase of the PN sequence. In the modulation process, the transmitter changes shifting length artificially, and the receiver can recover this shifting length through the peak position of the correlation function so as to transmit useful information [31].

The cyclic shifting operation does not change the spectrum spreading capability of the PN sequence. However, after this cyclic shifting operation, the synchronization function of the PN sequence is sacrificed. Therefore, a pilot channel is needed to provide the synchronization capability. A spreading sequence of the same family as the data channel is used in the pilot channel. The pilot channel works in traditional DSSS communications mode without any shifting process. The PN sequence of the data channel is cyclically shifted before the modulation process, i.e., the code phase is changed, so that the cyclic shift length can be used to carry useful information. The bits of the data channel are completely synchronized with those of the pilot channel.

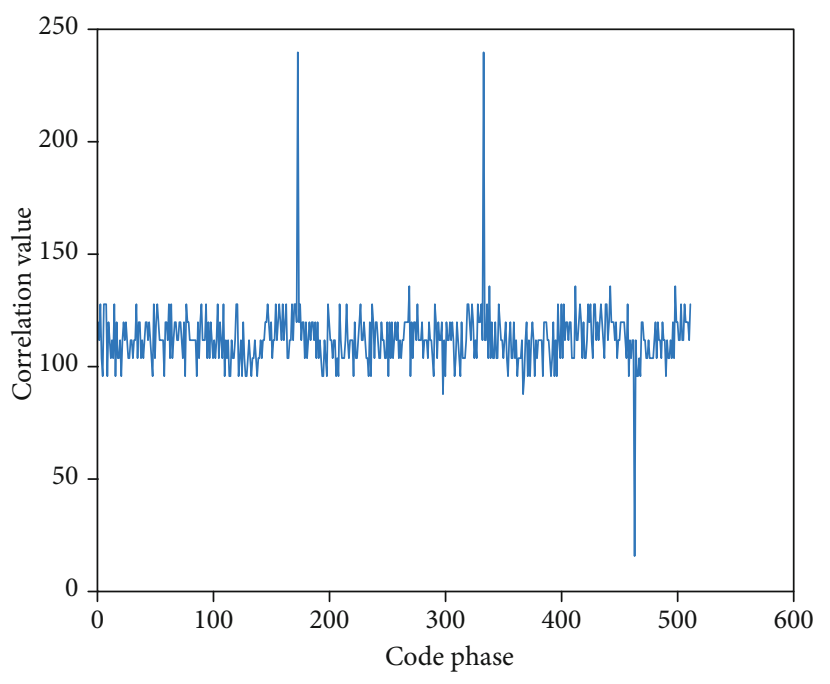

FIgURE 3: The correlation function of the CDIPPM signal: because the number of subdata channels is indefinite, the peak number of the correlation function is also indefinite.

PN sequence in the pilot channel carries binary information by bit reversing. In the data channel, an additional set of data information can be modulated by the cyclic shifting operation. The total state number of one symbol depends on the code length. The cyclic shifting operation brings more status to a PN sequence, which allows it to carry more information.

In this study, multiple spread spectrum signals are superimposed in one data channel to further increase the channel utilization rate. The PN sequence of every spread spectrum signal is the same, as shown in Figure 2. The hexagons in the figure represent a 7-chip length PN sequence, and the order of these chips is represented by a sequence number. $\mathrm{PN}$ sequence can be bit-reversed to carry binary information, which is denoted by $\mathrm{B} 0$ and $\mathrm{B} 1$. The data channels in the system, as illustrated in the figure, can carry 7 additional information states, denoted as D0-D6.

During the receiving process, the correlator would detect multiple peaks in correlation functions, as shown in Figure 3. 


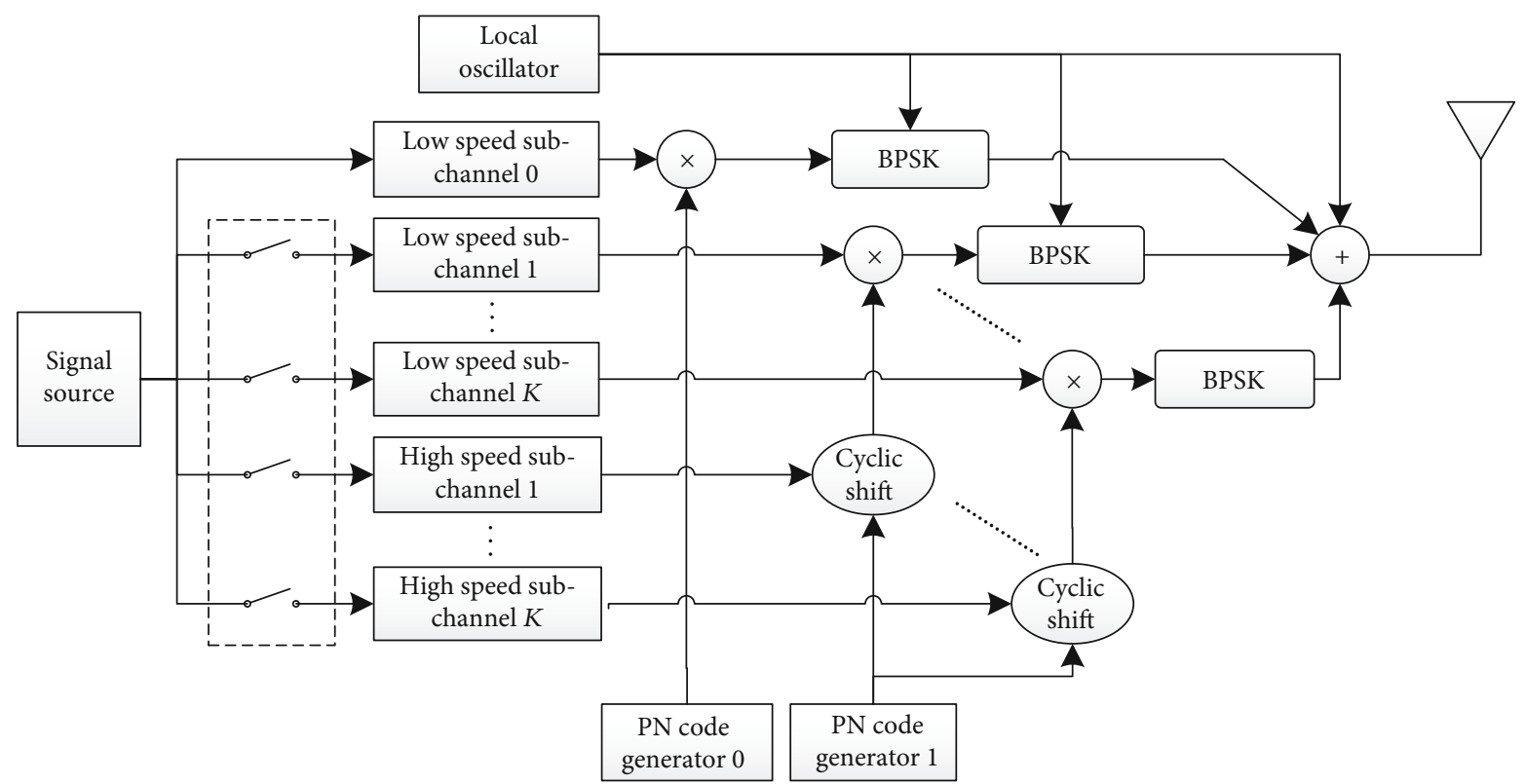

FIGURE 4: Modulation strategy of CDIPPM signal.

These peaks can be identified by the gross error removal theory. As mentioned before, the number of states of a PN sequence is $N_{c}$, which is the code length. When $M$ spread spectrum signals, with the same PN sequence, are superimposed together, the amount of states of each PN sequence will reach $C_{N_{c}}^{M}$. If the number of the spread spectrum signals involved in the superimposition is not a fixed number, the number of states will be further increased to $\sum_{i=1}^{M} C_{N_{c}}^{i}$, thereby increasing the information transfer speed.

Carrying information through the cyclic shifting of the PN sequence is essentially pulse-position modulation on the correlation domain. The amount of pulses used in this technique is variable, so the technique proposed here is named CDIPPM.

It is noteworthy that CDIPPM is different from combining several independent Cyclic Code Shift Keying (CCSK) signals together [32], because in CDIPPM signal, all the subdata channels use the same PN sequence. But if several CCSK signals with the same PN sequence were combined independently, it is impossible to distinguish the correlation peaks by the corresponding CCSK signals. Also, if two or more correlation peaks of different CCSK signals overlapped together, it is hard to distinguish them from one single correlation peak.

As the information of CDIPPM signal is transferred via time delay, this technique is more sensitive to multipath phenomenon. However, since the application of the satellite navigation and communication service is a satellite-ground or intersatellite link and the multipath phenomenon of such links is weak and suppressible by the directional antenna, the multipath phenomenon can be ignored in this application.

It is conceivable that the pilot channels can be designed like those in conventional navigation satellite signals in the downlink or in the intersatellite positioning and communication link of the SNCI system. The pilot channel still provides traditional, fully backward compatible satellite navigation

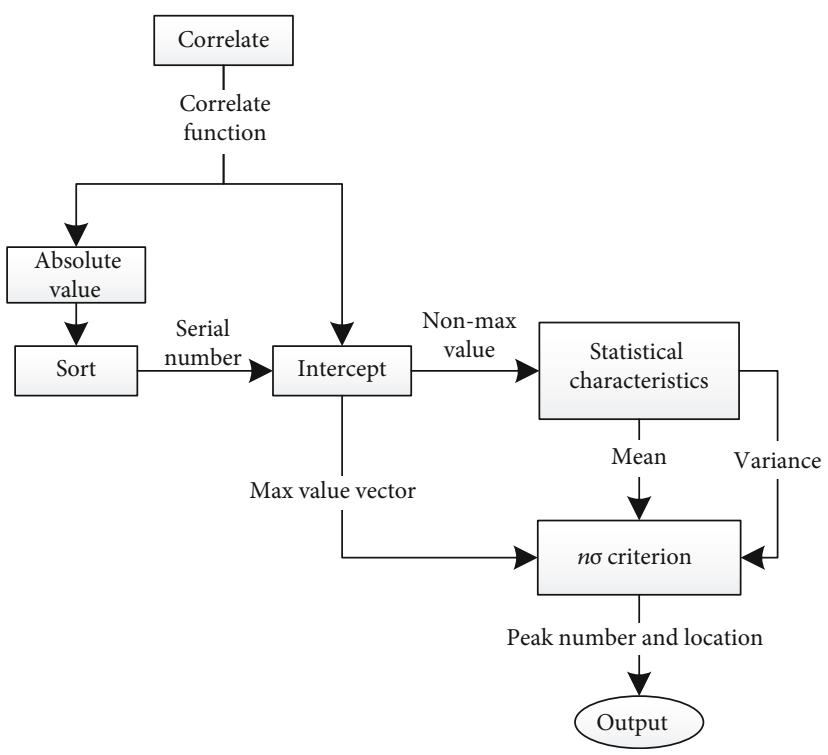

FIgURE 5: Peak detecting strategy of CDIPPM signal.

services, so the traditional navigation receiver can still maintain its positioning functions without modification. The data channel uses a similar signal system to provide higher-speed communication services. Under the general trend of software-based receivers, the receivers can be upgraded without significant change in the hardware, which is beneficial to the popularization of this technique.

\section{Signal Transceiver Strategy}

3.1. Modulation. The CDIPPM-based SNCI system is similar to the traditional GNSS in signal modulation. The traditional GNSS signal is directly utilized as the pilot channel, while in 


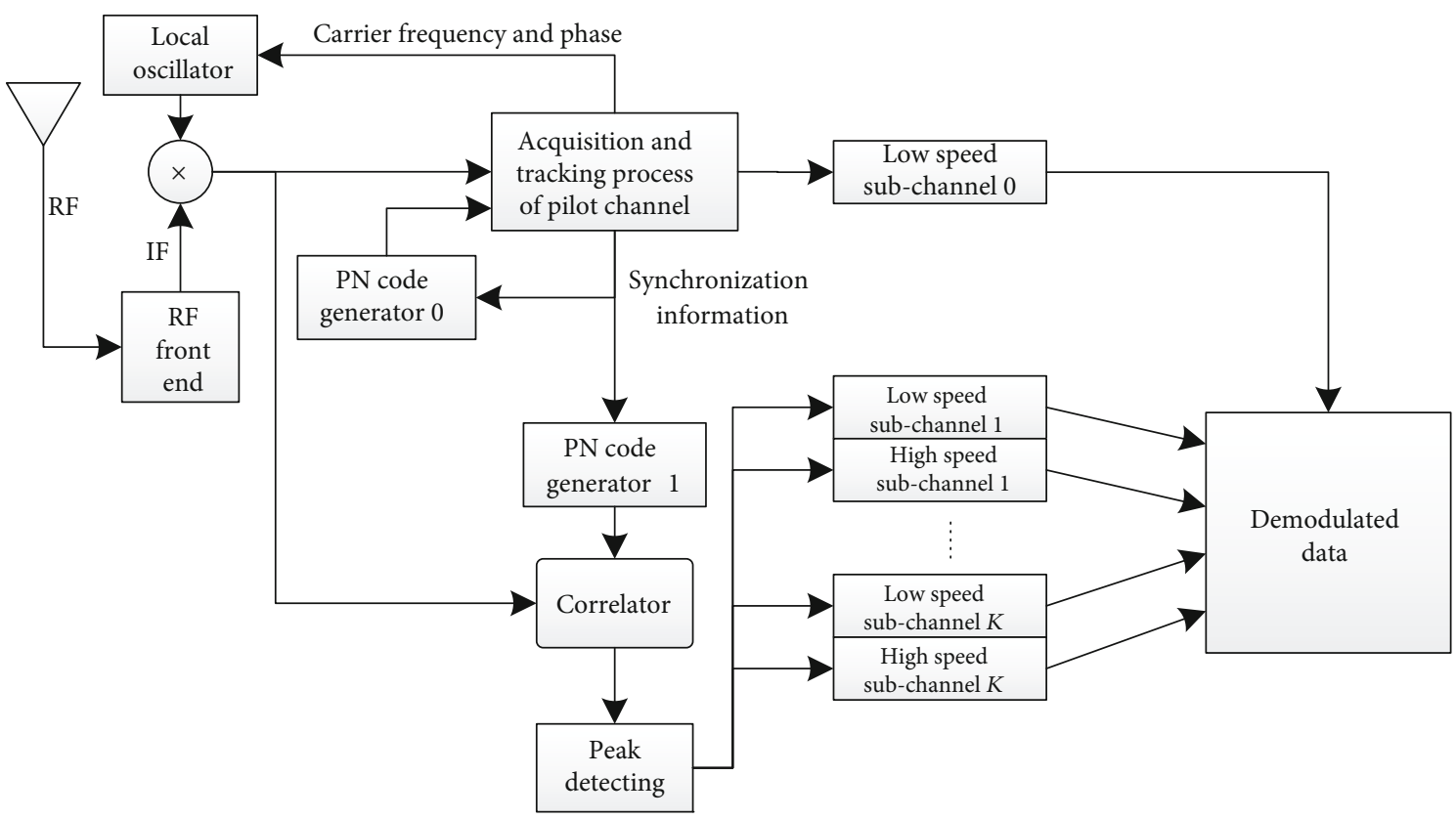

Figure 6: Demodulation strategy of CDIPPM signal.

the data channel only a cyclic shift process is added to the modulation scheme as Figure 4.

The "low speed subchannel 0" stands for the pilot channel, and the "low speed subchannel 1" to "low speed subchannel $K$ " are used to control the flip of the peaks. The "high speed subchannels" are used to control the shifting process of the PN code. The information rate ratio between low speed subchannels and high speed subchannels is $1+R:(1 / K) \sum_{R=1}^{K} \log _{2} C_{N_{c}}^{R}$.

3.2. Demodulation. For the receiver, as a traditional DSSS signal is used as the pilot signal, the demodulation algorithm of the pilot channel is the same as that of the traditional GNSS signal, including acquisition and tracking processes. Due to the cyclic shift operation applied on the PN code, the data channel loses its self-synchronization capability; the pilot channel is needed to provide synchronization for the data channel. The local PN sequence generator of the data channel should be feedback controlled by the tracking loop of the pilot channel.

Since the PN code of the data channel is cyclically shifted, the correlation peak of each cycle is always changing, disabling the delay locked loop of the tracking process. Therefore, for the data channel, the tracking process is altered from the receiving algorithm. For the same reason, the serial correlation algorithm is unavailable in data channel demodulation. Conversely, the position of the correlation peak can be calculated within one cycle of PN code by utilizing the frequency domain correlation algorithm. Thus, the frequency domain correlation algorithm is utilized for CDIPPM signal demodulation.

On the other hand, in the data channel, the number of correlation peaks is indefinite. Therefore, a peak detection strategy is needed after the correlator to determine the number of peaks. Since the correlation function contains enough samples and the maximum number of peaks is known, $3 \sigma$ criterion is used for peak number detection. But here, we
TABLE 1: The transmit speed comparison of reformed GNSS signals $(K=3)$.

\begin{tabular}{lcccc}
\hline Signal & $\begin{array}{c}\text { PN } \\
\text { code } \\
\text { length }\end{array}$ & $\begin{array}{c}\text { Chip } \\
\text { rates } \\
\text { (cps) }\end{array}$ & $\begin{array}{c}\text { Speed of } \\
\text { traditional } \\
\text { signal (bps) }\end{array}$ & $\begin{array}{c}\text { Speed of } \\
\text { reformed signal } \\
\text { (bps) }\end{array}$ \\
\hline GPS L1 & 1023 & $1.023 \mathrm{M}$ & 50 & $30.4587 \mathrm{~K}$ \\
BeiDou B1 & 2046 & $2.046 \mathrm{M}$ & 50 & $31.8248 \mathrm{~K}$ \\
GLONASS & 511 & $0.511 \mathrm{M}$ & 50 & $25.8174 \mathrm{~K}$ \\
L1 & & & 250 & $9.3526 \mathrm{~K}$ \\
Galileo E1 & 4092 & $1.023 \mathrm{M}$ & 250 \\
\hline
\end{tabular}

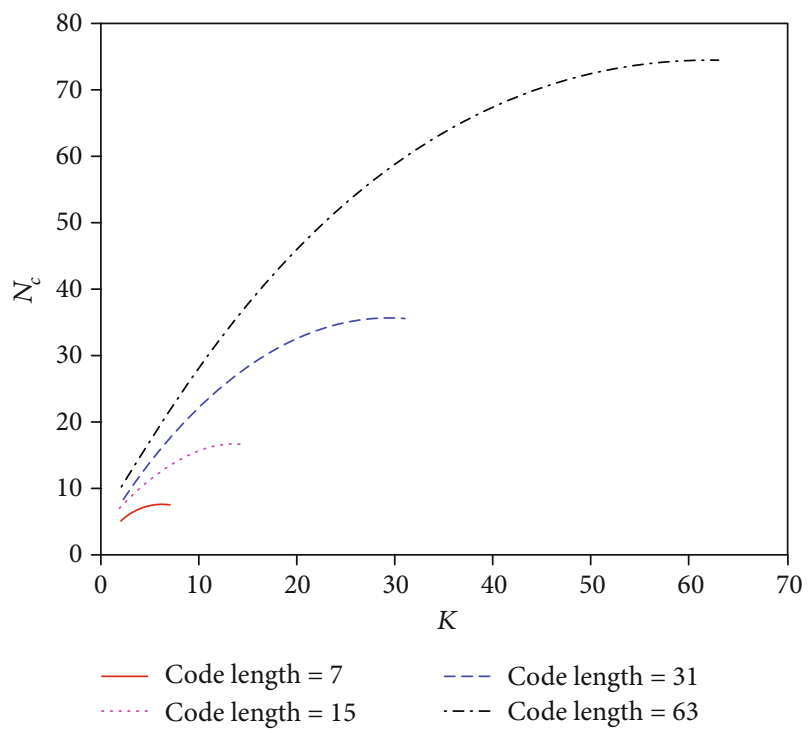

Figure 7: The relationship between $N_{c}$ and $K$. 
TABLE 2: Ideal $K$ values correspond to different $N_{c \text {. }}$

\begin{tabular}{lccccccccc}
\hline$N_{c}$ & 3 & 7 & 15 & 31 & 63 & 127 & 255 & 511 & 1023 \\
$K$ & 1 & 6 & 13 & 29 & 56 & 126 & 250 & 498 & 994 \\
$N_{c}$ & 2047 & 4095 & 8191 & 16383 & 32767 & 65535 & 131071 & 262143 \\
$K$ & 1986 & 7938 & 15874 & 31746 & 63490 & 126978 & 253954 & 507906 & 1015810 \\
\hline
\end{tabular}

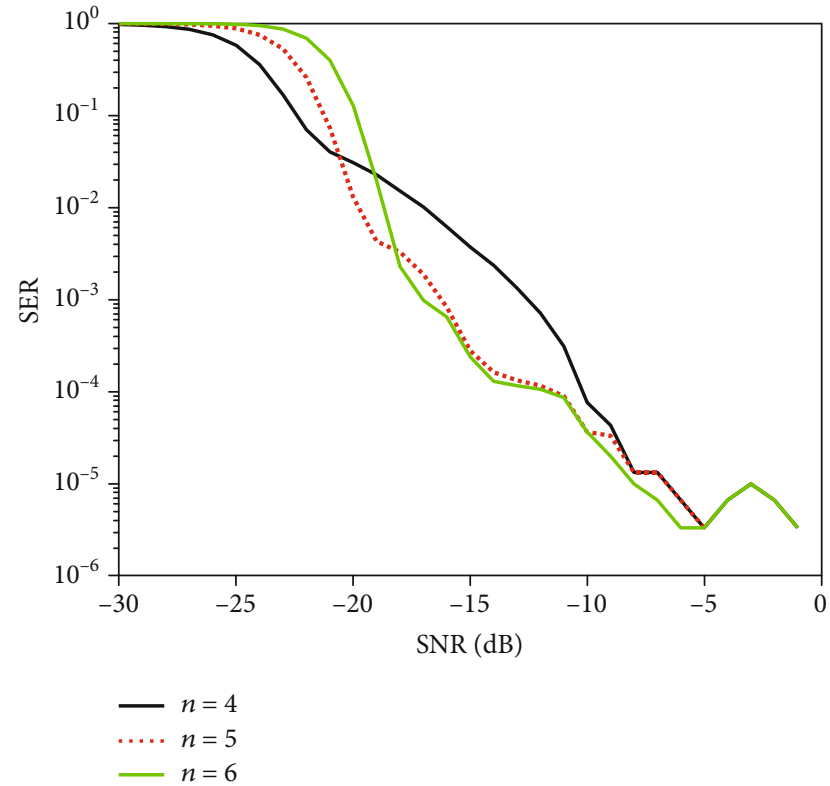

FIgURE 8: Error feature simulation. For the solid line, $n=4$; for the dotted line, $n=5$; for the chain line, $n=6$.

make two changes to $3 \sigma$ criterion. Firstly, $n \sigma$ is used as the threshold instead of $3 \sigma$, because the peak number is too small to the total sample number for one PN code. Secondly, several largest samples in absolute value could be altered when calculating the standard deviation. Thus, the influence of the peak value on the statistical characteristics can be avoided. The peak detection process is shown in Figure 5. In summary, the receiving algorithm of CDIPPM is shown in Figure 6. Figure 6 illustrates that the signal detection strategy of CDIPPM includes the detection strategy of the traditional GNSS signal so that a CDIPPM receiver can be easily converted from a conventional GNSS receiver.

3.3. Channel Utilization Analysis. For traditional Binary Phase Shift Keying (BPSK) signals, the channel utilization can be calculated by the Shannon formula. However, in the case of a conventional DSSS signal under BPSK modulation, one bit is represented by a PN sequence with a code length of $N_{c}$. Thus, when the energy of one bit remains constant, the bandwidth $B_{N 1}$ of the signal is correspondingly extended $N_{c}$ times. So, the channel utilization rate becomes

$$
\frac{C}{B_{N 1}}=\frac{1}{N_{c}} \log _{2}\left(1+\frac{E_{b}}{N_{0}}\right),
$$

where $C$ is the channel capacity, $E_{b}$ illustrates the energy of one bit, and $N_{0}$ represents the noise power density.However,

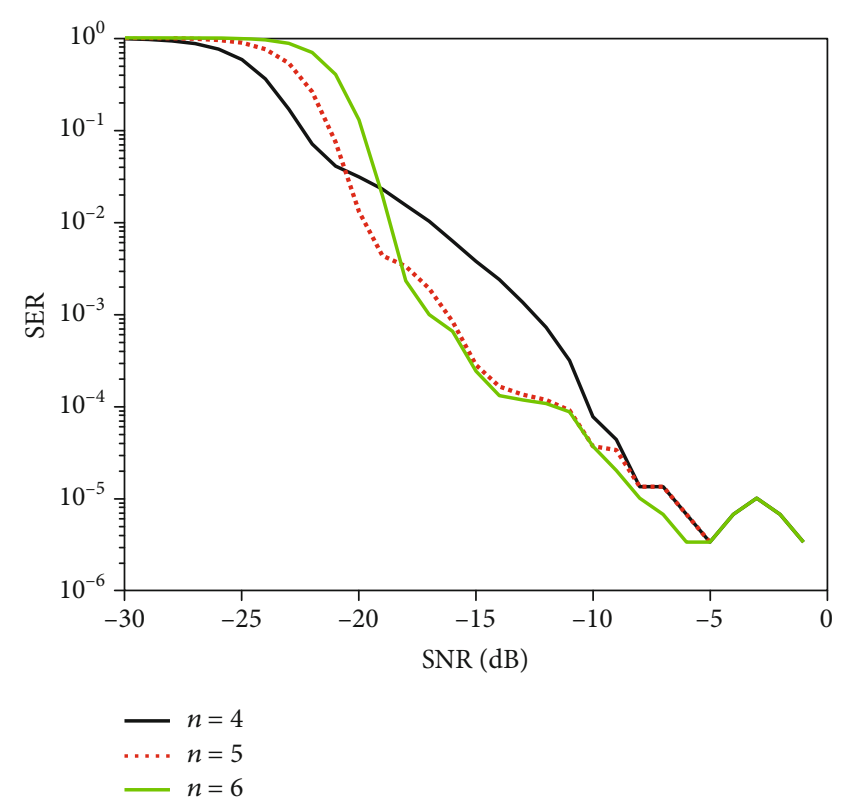

FIGURE 9: Error feature simulation (total peak number is 1). For the solid line, $n=4$; for the dotted line, $n=5$; for the chain line, $n=6$.

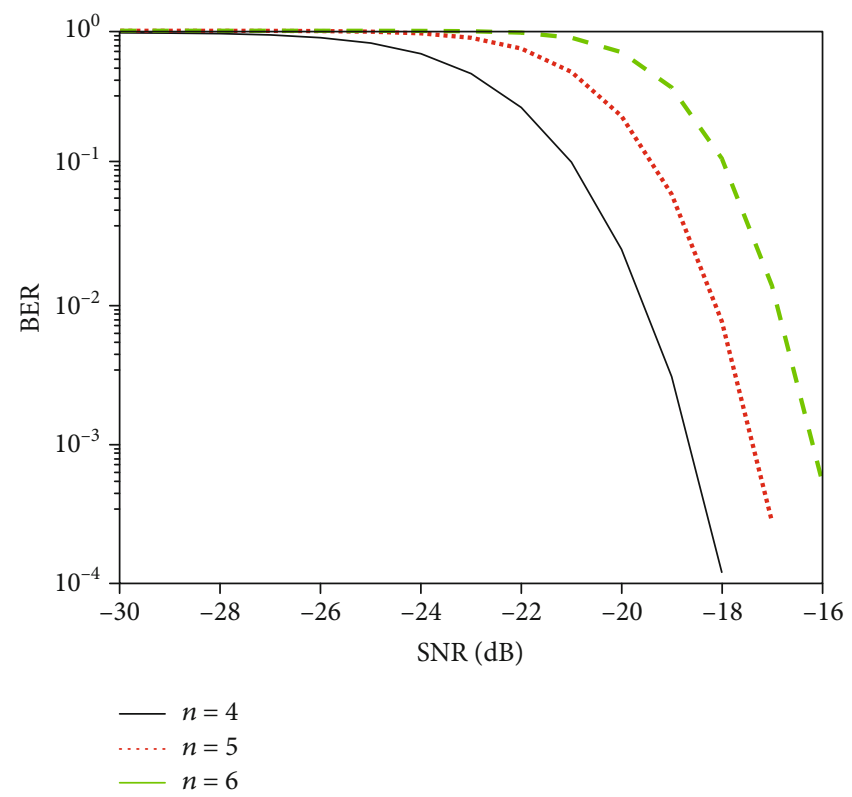

FIgURE 10: Error feature simulation for CDMA signal. For the solid line, $n=4$; for the dotted line, $n=5$; for the chain line, $n=6$.

if information is transmitted through the correlation peak position, since the energy of one bit remains the same in the horizontal comparison while the amount of information 


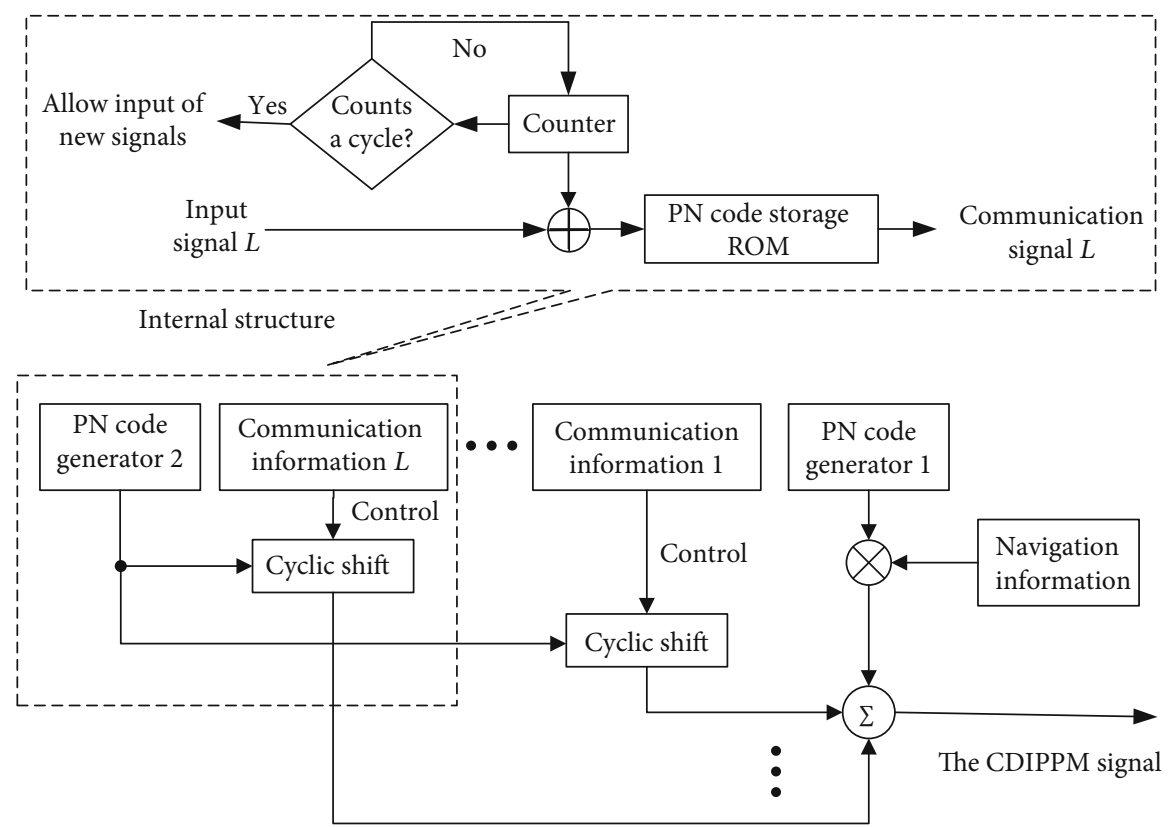

Figure 11: The signal generator: the dotted line is the structure of the communication signal $L$.

transmitted in a PN code period is increased from one bit to $1+\log _{2} N_{c}$ bit, the period occupied by a PN code should correspondingly expand $1+\log _{2} N_{c}$ times to ensure that the energy of one bit is unchanged. So, its bandwidth $B_{N 2}$ becomes

$$
B_{N 2}=\frac{B_{N 1}}{1+\log _{2} N_{c}},
$$

and the channel utilization rate can be expressed as

$$
\frac{C}{B_{N 2}}=\frac{1+\log _{2} N_{c}}{N_{c}} \log _{2}\left(1+\frac{E_{b}}{N_{0}}\right) \text {. }
$$

Furthermore, if the number of correlation peaks $R$ is between 1 and $K$, the amount of information that can be transferred per code period is also correspondingly increased to $R+\log _{2} C_{N_{c}}^{R}$ bit. The corresponding signal energy is $R$, so the channel utilization can be expressed as

$$
\frac{C}{B_{R}}=\frac{R+\log _{2} C_{N_{c}}^{R}}{N_{c}} \log _{2}\left(1+\frac{E_{b}}{N_{0}}\right),
$$

where $B_{R}$ is the normalized signal bandwidth. For the CDIPPM transmission system, assuming that the occurrence probabilities of each symbol are the same, the overall channel utilization rate for a system with up to $K$ peaks can be expressed as

$$
\frac{C}{B}=\frac{1}{K} \sum_{R=1}^{K} \frac{R+\log _{2} C_{N_{c}}^{R}}{R N_{c}} \log _{2}\left(1+\frac{E_{b}}{N_{0}}\right)
$$

It can be seen that the channel utilization of the CDIPPM signal is improved when compared with that of the conven- tional DSSS signal.Although its channel utilization rate cannot exceed the BPSK signal, signal power is reduced due to the processing gain. The multiple access capability of DSSS signal also ensures the overall network rate. Assuming the system bandwidth is constant and both navigation and communication services are needed, neither the frequency division mode nor the time division mode can make full use of bandwidth resources and may require users to equip both kinds of receivers at the same time. The CDIPPM signal is similar to the traditional DSSS signal, which is also used as the pilot channel and provides traditional navigation services. It can provide both navigation and highspeed communication services while ensuring that users occupy all resources and that the receiving terminals guarantee consistency.For example, the GPS L1 signal, BeiDou B1 signal, GLONASS L1 signal, and Galileo E1 signal are widely used for civil applications. If these signals are reformed by CDIPPM signal, the transmit speed would be as shown in Table 1.

Here, we introduce a transmission rate increase factor $I$ $\left(K, N_{c}\right)$, to describe the transmission rate increment of a traditional CDMA signal if it was changed to CDIPPM signal. The factor $I\left(K, N_{c}\right)$ is calculated as

$$
I\left(K, N_{c}\right)=\frac{1}{K} \sum_{R=1}^{K}\left(R+\log _{2} C_{N_{c}}^{R}\right)
$$

The relationship between $N_{c}$ and $K$ is shown in Figure 7.It can be seen that $N_{c}$ and the best $K$, which makes the function $I\left(K, N_{c}\right)$ maximum, is not a monotonic relationship, because bigger $K$ value brings more states to one symbol by bit flipping. But $C_{N_{c}}^{K}$ and $C_{N_{c}}^{N_{c}-K}$ pervade the same state numbers that means bigger $K$ value brings fewer states to one symbol, by 


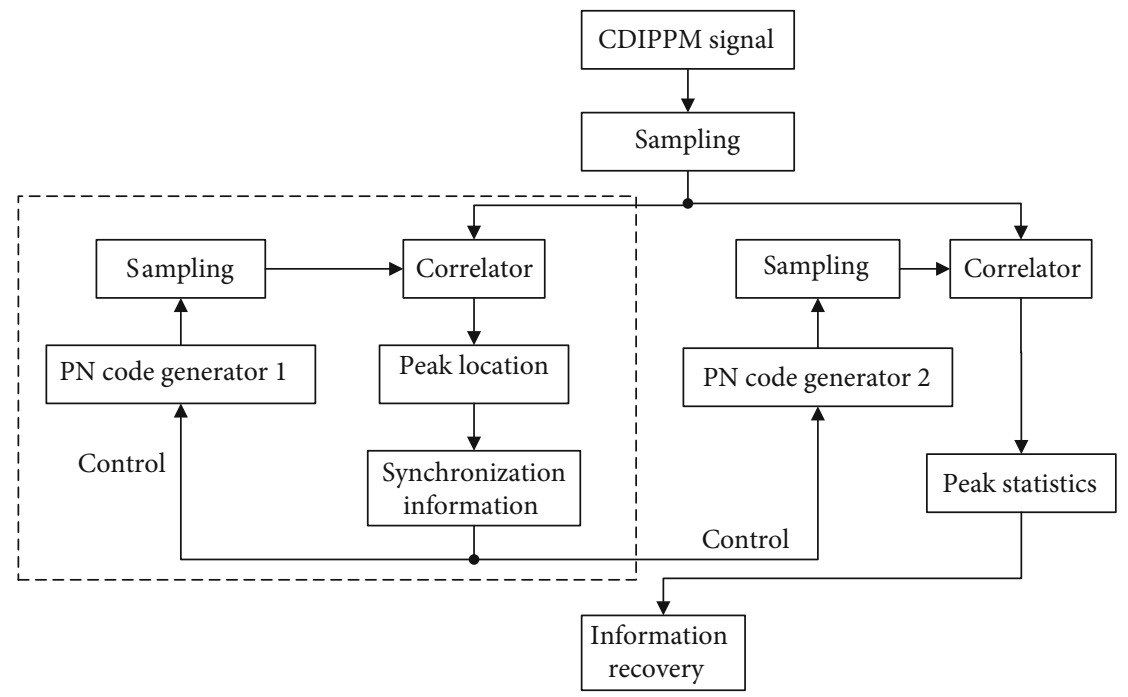

FIGURE 12: The signal demodulator.

pulse positions. Based on Equation (6), the best $K$ for a certain $N_{c}$ is the positive integer that most fulfills

$$
\min \left(\frac{1}{2}+\frac{1}{K(K-1)} \log _{2} \prod_{i=1}^{K} \frac{\left(N_{c}-i+1\right)^{(i-1)}}{i^{(i-1)}}\right)
$$

The analytical solution of $K$ is too complex to calculate; here, we give the ideal $K$ values correspond to different $N_{c}$ in Table 2. Notice that some of the speed increases in Table 2 mismatch with Equation (6); that is because one bit of GPS L1 signal and BeiDou B1 signal is formed by 20 PN sequences. Also, this ideal $K$ value is only a theoretical result. In the system design process, the error characteristics should also be taken into consideration, because bigger $K$ increases the complexity and self-relation side-lobe interference of the system.

\section{Error Probability Analysis}

The noise of correlation function corresponds to the Rayleigh distribution, as

$$
f_{n}(v)=\frac{v}{\sigma_{n}^{2}} e^{-v^{2} / 2 \sigma_{n}^{2}}
$$

where $v$ represents the correlation value and $\sigma_{n}$ stands for the standard deviation of the noise. In the fully synchronized status, each correlation peak is in line with the Rice distribution, expressed by

$$
f_{s}(v)=\frac{v}{\sigma_{n}^{2}} e^{-\left(v^{2}+A^{2}\right) / 2 \sigma_{n}^{2}} I_{0}\left(\frac{v A}{\sigma_{n}^{2}}\right),
$$

where $A$ is the signal amplitude and $I_{0}$ is the first-order Bessel function. For any $s \neq 0$, when $R_{a}(s)>R_{a}(0)$, a symbol error occurs, where $R_{a}(x)$ stands for the $x$-th value of the autocor- relation function. Therefore, the Symbol Error Rate (SER) of one peak should be expressed as

$$
\begin{aligned}
P_{\mathrm{SER}} & =\int_{0}^{\infty} f_{n}(y) \int_{0}^{y} f_{s}(x) d x d y \\
& =0.5-\int_{0}^{\infty} \frac{y}{\sigma_{n}^{2}} e^{-y^{2} / 2 \sigma_{n}^{2}} Q_{1}\left(\frac{A}{\sigma_{n}}, \frac{y}{\sigma_{n}}\right) d y,
\end{aligned}
$$

where $Q_{1}$ is the Marcum $Q$-function. When $R$ subdata were integrated together, the variance of the correlation function would be increased to $R \sigma_{n}^{2}$. When the number of peaks $R$ is undefined, assuming that the occurrence probabilities of each symbol are the same, the overall error rate can be expressed as

$P_{\mathrm{SER}}^{K}=0.5-\frac{\sum_{R=1}^{K} \int_{0}^{\infty}\left(y / R \sigma_{n}^{2}\right) e^{-y^{2} / 2 R \sigma_{n}^{2}} Q_{1}\left(A / \sqrt{R} \sigma_{n}, y / \sqrt{R} \sigma_{n}\right) d y}{K}$,

where $K$ is the total peak number of the system. To verify the error characteristics of CDIPPM signals, corresponding simulations were carried out using a Gaussian channel. The simulation uses a Gold sequence with a code length of 1023 as a spreading sequence with an intermediate frequency of 4.096 $\mathrm{MHz}$. The maximum correlation peak number is 3. Since each PN code period should be considered as a unitary symbol in the transmission, the commonly used Bit Error Rate (BER) can be replaced by the SER, both of which are essentially the same. For each symbol, an error in either the peak number, the peak position, or the bit polarity is regarded as a transmission error. Figure 8 shows the simulation results; different $n$ values are used for peak detection strategy.

For comparison, a set of similar systems is simulated, in which the total peak number is 1 , which corresponds to the CCSK signal. The simulation results are shown in Figure 9.

Similarly, in this simulation, the system characters are the same with the CDIPPM system, and SER is used instead of 


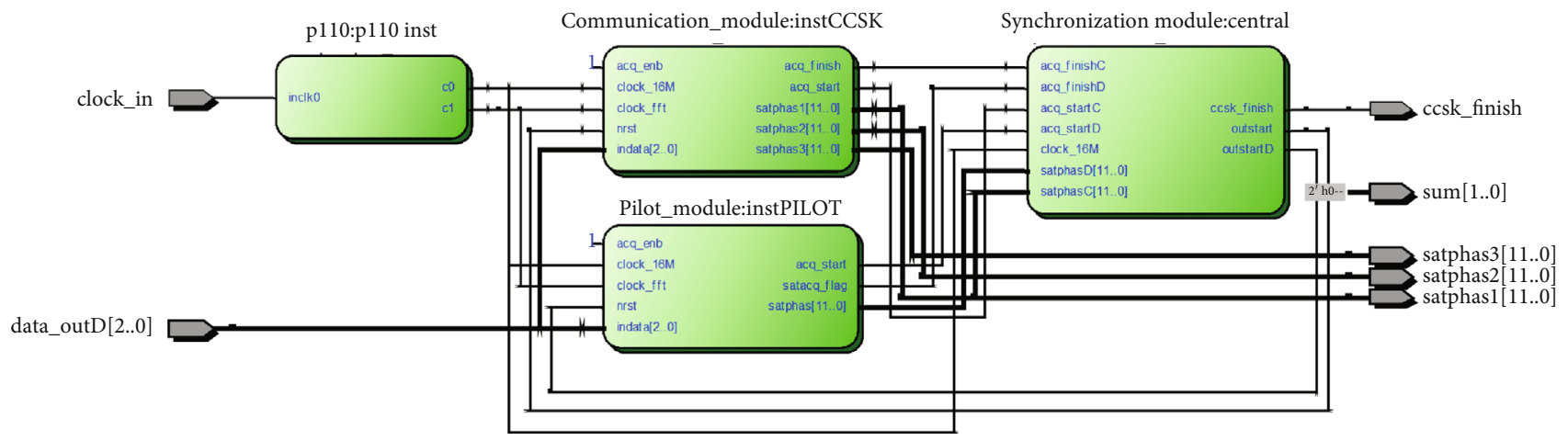

FIgURE 13: The signal demodulator RTL diagram.

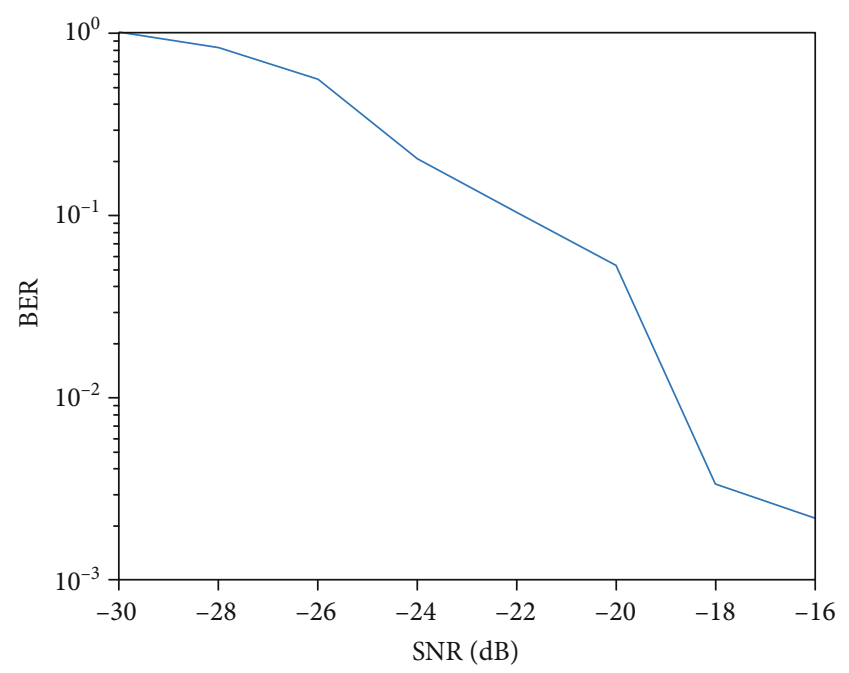

FIGURE 14: Error feature experiment for CDMA signal.

BER. Also, corresponding CDMA systems are simulated with the similar system characters. The simulation results are shown in Figure 10.

For the sake of fairness, the same detection strategy is used in these 3 simulations. It can be seen from the figures that the reliability performance of CDIPPM system is $8 \sim 10$ $\mathrm{dB}$ 's lower than the traditional systems; this is the cost of improving the effectiveness. And in the aforementioned systems, the data rate is $1 \mathrm{Kbps}$ for the CDMA system, 10.9986 Kbps for the CCSK system, and $30.4087 \mathrm{Kbps}$ for the CDIPPM system $(K=3)$.

\section{Hardware Implementation}

In this paper, the baseband transmission practicability of the CDIPPM signal is verified on FPGA platforms. In order to realize the signal modulation strategy in Figure 5, the signal generator is designed as shown in Figure 11. The CDIPPM signal is composed by a pilot channel and a data channel. The pilot channel is generated as the traditional GNSS signal. The data channel is generated by accumulating L CCSK signals, where $L$ is determined by the communication data. The CCSK signal is generated by cyclically shifting the local $\mathrm{PN}$ code, which is marked by the dotted line. The local PN

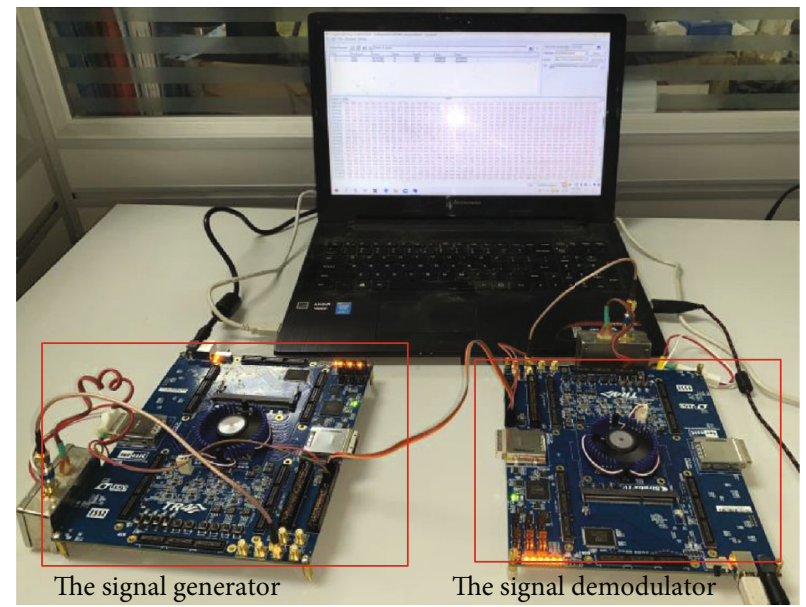

FIGURE 15: The hardware platform.

code is stored in a ROM, and the read address is generated by a loop counter; the initial phase of the loop counter is also determined by the communication data. The signal demodulator circuit is designed as Figure 12. The signal synchronization is obtained by the pilot signal acquisition process, which controls the local PN code generator. As marked by the dotted line, the pilot signal acquisition is similar with GNSS acquisition strategy. The structure of the data channel receiver is similar to that of the pilot channel acquisition circuit; the only difference is the peak value detector. This detector selects $L$ maximum correlation values and sorts them in descending order. If a maximum value exceeds the threshold, the peak position would be recorded as the information; otherwise, it is regarded as noise interference. The signal demodulator RTL diagram based on FPGA is shown in Figure 13, where "instPILOT" part is the pilot signal acquisition circuit, "instCCSK" is the data channel receiver circuit, and "central" is the core controller circuit. The interaction between the "instPILOT" and the "instCCSK" and the synchronization of signals is completed by the core controller.

To verify the error characteristics of CDIPPM signals, corresponding experiments were carried out using a Gaussian channel. Figure 14 shows the experimental results. The experiments use a Gold sequence with a code length of 1023 as a spreading sequence with an intermediate frequency of $4.096 \mathrm{MHz}$. The maximum correlation peak number is 3 . 
The hardware platform uses Altera's Stratix IV, and the clock uses a $16.368 \mathrm{MHz}$ temperature-compensated crystal oscillator. The platform of the baseband communication experiment is shown in Figure 15, where the left side is the signal generator and the right side is the signal demodulator. In order to verify the performance of the system, the demodulated information was stored in an in-system memory and compared with the transmitted data on the PC.

\section{Conclusions}

The SNCI is an important part of IoT. In light of the bottleneck of SNCI systems, the CDIPPM technology proposed in this paper carries information by the combination of pulse position in correlation domain. While maintaining the spread spectrum gain and the multiple access performance, the information transmission rate of traditional navigation signals is enhanced. This makes the signal compatible with navigation and communication features. The quantity of the enhancement depends on the total peak number and the PN code length of the system. Take GPS for example, CDIPPM technology can enhance the data rate from 50 bps to $30.4587 \mathrm{Kbps}$, when the total peak number is 3. In the future, the source coding problem, multipath problem, and the timing method will be the following research direction of CDIPPM technology.

\section{Data Availability}

No data were used to support this study.

\section{Conflicts of Interest}

The authors declare that there is no conflict of interest regarding the publication of this paper.

\section{Acknowledgments}

Many thanks are due to Mr. Craig Noles who provided professional language assistance for this paper. In memory of my grandfather who has passed away when this paper was first ready to submit, his wisdom and foresight changed the fate of the entire family. This research is supported by the National Natural Science Foundation of China (Youth), project number 61701072.

\section{References}

[1] D. Gaudenzi, "Payload nonlinearity impact on the Globalstar forward link multiplex. I. Physical layer analysis," IEEE Transactions on Vehicular Technology, vol. 48, no. 3, pp. 960-976, 1999.

[2] F. J. Dietrich, P. Metzen, and P. Monte, "The Globalstar cellular satellite system," IEEE Transactions on Antennas and Propagation, vol. 46, no. 6, pp. 935-942, 1998.

[3] M. A. Mujeebu, "The disappearance of MH370 and the search operations - the role of technology and emerging research challenges," IEEE Aerospace and Electronic Systems Magazine, vol. 31, no. 3, pp. 6-16, 2016.
[4] J. Chen, J. Ma, N. Zhong et al., "WaaS: wisdom as a service," IEEE Intelligent Systems, vol. 29, no. 6, pp. 40-47, 2014.

[5] Federal Aviation Administration, Global Positioning System Wide Area Augmentation System (WAAS) Performance Standard, Federal Aviation Administration, 2008.

[6] G. Grunwald, M. Bakuła, A. Ciećko, and R. Kaźmierczak, "Examination of GPS/EGNOS integrity in north-eastern Poland," IET Radar, Sonar and Navigation, vol. 10, no. 1, pp. 114-121, 2016.

[7] H. Manabe, "Status of MSAS: MTSAT satellite based augmentation system," in Proceedings of the 21st International Technical Meeting of the Satellite Division of The Institute of Navigation (ION GNSS 2008), pp. 1032-1059, Savannah, GA, 2008.

[8] Y.-F. Tsai and K.-S. Low, "Performance assessment on expanding SBAS service areas of GAGAN and MSAS to Singapore region," in Proceedings of IEEE/ION PLANS 2014, pp. 686-691, Monterey, CA, 2014.

[9] T. Suzuki and N. Kubo, "Precise point positioning for mobile robots using software GNSS receiver and QZSS LEX signal," in 2013 IEEE/RSJ International Conference on Intelligent Robots and Systems, pp. 369-375, Tokyo, Japan, 2013.

[10] H. Hoang-Van, T. N. T. Thanh, and V. La The, "An evaluation of precise point positioning using QZSS LEX signal in Vietnam," in 2016 3rd National Foundation for Science and Technology Development Conference on Information and Computer Science (NICS), pp. 234-239, Danang, Vietnam, 2016.

[11] A. D. Sarma, N. Prasad, and T. Madhu, "Investigation of suitability of grid-based ionospheric models for GAGAN," Electronics Letters, vol. 42, no. 8, pp. 478-479, 2006.

[12] M. Karthick and K. Supriya, "Design of multiband fractal antenna for IRNSS and GAGAN applications," in 2015 International Conference on Innovations in Information, Embedded and Communication Systems (ICIIECS), pp. 1-4, Coimbatore, India, 2015.

[13] A. S. Ganeshan, GAGAN, Second Meeting of the International Committee on Global Navigation Satellite System (ICG-02), Bangalore, India, 2007.

[14] S. Marila, M. Z. H. Bhuiyan, J. Kuokkanen, H. Koivula, and H. Kuusniemi, "Performance comparison of differential GNSS, EGNOS and SDCM in different user scenarios in Finland," in 2016 European Navigation Conference (ENC), pp. 1-7, Helsinki, Finland, 2016.

[15] Z. Wang, S. Fang, S. Fu, and S. Jia, “An Inmarsat BGAN terminal patch antenna array with unequal input impedance elements and conductor-backed ACPW series-feed network," IEEE Transactions on Antennas and Propagation, vol. 60, no. 3, pp. 1642-1647, 2012.

[16] X. Lyu, A. Stove, M. Gashinova, and M. Cherniakov, "Ambiguity function of Inmarsat BGAN signal for radar application," Electronics Letters, vol. 52, no. 18, pp. 1557-1559, 2016.

[17] G. X. Ai, H. L. Shi, and H. T. Wu, "Positioning system based satellite communication and Chinese area positioning system," China Astons Atrophies, vol. 8, pp. 44-47, 2008.

[18] C. Jun-xia, S. Hu-li, and P. Jun, "A novel low-rate satellite communication system based on SIGSO satellite," in 2009 International Conference on Information Management and Engineering, pp. 519-522, Kuala Lumpur, Malaysia, 2009.

[19] J. Zhang, H.-c. Yang, and D. Yang, "Design of a new high-gain circularly polarized antenna for Inmarsat communications," 
IEEE Antennas and Wireless Propagation Letters, vol. 11, pp. 350-353, 2012.

[20] Dan Yang, Hong-chun Yang, Jing Zhang, and Yang Li, "A novel circularly polarized bowtie antenna for Inmarsat communications [antenna applications corner]," IEEE Antennas and Propagation Magazine, vol. 54, no. 4, pp. 317-325, 2012.

[21] Committee of Experts on the field of aerospace, The Use of Iridium Achieved Significant Progress to Improve GNSS Capabilities, 863 Aerospace Technology News, 2009.

[22] S. Han, Y. Zhang, W. Meng, C. Li, and Z. Zhang, "Full-duplex relay-assisted macrocell with millimeter wave backhauls: framework and prospects," IEEE Network, vol. 33, no. 5, pp. 190-197, 2019.

[23] S. Han, Y. Huang, W. Meng, C. Li, N. Xu, and D. Chen, "Optimal power allocation for SCMA downlink systems based on maximum capacity," IEEE Transactions on Communications, vol. 67, no. 2, pp. 1480-1489, 2019.

[24] S. Han, Y. Li, W. Meng, C. Li, T. Liu, and Y. Zhang, "Indoor localization with a single $\mathrm{Wi}$-Fi access point based on OFDM-MIMO," IEEE Systems Journal, vol. 13, no. 1, pp. 964-972, 2019.

[25] S. Han, L. Chen, W. Meng, and C. Li, "Improve the security of GNSS receivers through spoofing mitigation," IEEE Access, vol. 5, pp. 21057-21069, 2017.

[26] S. Han, Z. Gong, W. Meng, C. Li, and X. Gu, "Future alternative positioning, navigation, and timing techniques: a survey," IEEE Wireless Communications Magazine, vol. 23, no. 6, pp. 154-160, 2016.

[27] S. Han, D. Luo, W. Meng, and C. Li, "Antispoofing RAIM for dual-recursion particle filter of GNSS calculation," IEEE Transactions on Aerospace and Electronic Systems, vol. 52, no. 2, pp. 836-851, 2016.

[28] X. Liu and X. Zhang, "NOMA-based resource allocation for cluster-based cognitive industrial Internet of things," IEEE Transactions on Industrial Informatics, vol. 16, no. 8, pp. 5379-5388, 2020.

[29] X. Liu, X. B. Zhai, W. Lu, and C. Wu, "QoS-guarantee resource allocation for multibeam satellite industrial Internet of things with NOMA," IEEE Transactions on Industrial Informatics, vol. 17, no. 3, pp. 2052-2061, 2021.

[30] X. Liu and X. Zhang, "Rate and energy efficiency improvements for 5G-based IoT with simultaneous transfer," IEEE Internet of Things Journal, vol. 6, no. 4, pp. 5971-5980, 2019.

[31] L. Xin, J. Min, and N. Zhenyu, "Multi-modal cooperative spectrum sensing based on Dempster-Shafer fusion in 5G-based cognitive radio,” IEEE Access, vol. 6, pp. 199-208, 2018.

[32] A. Y. Wong and V. C. M. Leung, "Code-phase-shift keying: a power and bandwidth efficient spread spectrum signaling technique for wireless local area network applications," CCECE '97. Canadian Conference on Electrical and Computer Engineering. Engineering Innovation, vol. 2, pp. 478-481, 1977. 